\title{
Tuberculous Granuloma of Thyroid Gland Mimicking Metastatic Thyroid Carcinoma: A Case Report and Review of Literature
}

\author{
Ahmet Görgel ${ }^{1}$ (D), Şenol Fatih Elbir ${ }^{2}$ (D) Erkan Karataş $^{3}$ (D), Arzu Avcı ${ }^{4}$ (i) \\ ${ }^{1}$ Gozde Academy Hospital, Department of Endocrinology and Metabolism, Malatya, Turkey \\ ${ }^{2}$ Gozde Academy Hospital, Department of Radiodiagnostic, Malatya, Turkey \\ ${ }^{3}$ Gozde Academy Hospital, Department of Otorhinolaryngology, Malatya, Turkey \\ ${ }^{4}$ Izmir Katip Celebi University, Ataturk Training and Research Hospital, Faculty of Medicine, Department of Pathology, Izmir, Turkey
}

ORCID ID: A.G. 0000-0002-7766-9525; Ş.F.E. 0000-0002-0446-9303; E.K. 0000-0002-9616-7168; A.A. 0000-0002-5522-0022

Citation: Gorgel A, Elbir SF, Karatas E, Avci A. Tuberculous granuloma of thyroid gland mimicking metastatic thyroid carcinoma: A case report and review of literature. Tr-ENT 2021;31(1):20-4. https://doi.org/10.26650/Tr-ENT.2021.52724

\section{ABSTRACT}

Tuberculosis is a problem to public health, especially in developing countries. However, involvement of the thyroid gland is rare. Herein, a 53-yearold woman with complaint of painless swelling on the right side of her neck is presented. Nodular goiter was detected in addition to multiple lymphadenopathies on the right cervical region. Tuberculosis was diagnosed based on histological findings in both the thyroid gland and cervical lymph nodes contrary to our expectation which was thyroid carcinoma.

Keywords: Thyroid Tuberculosis, Tuberculous Granuloma, Cervical Lymphadenopathy

\section{INTRODUCTION}

Tuberculosis is an infectious disease that has threatened humanity from ancient times to the present. The etiology of the disease was not understood until the end of $19^{\text {th }}$ century despite the fact that its contagious features had been known for many centuries. Mycobacterium tuberculosis, the bacteria that causes tuberculosis was discovered by Robert Koch in 1882. Tuberculosis mainly settles in the lung, however the rate of extrapulmonary tuberculosis has increased in the last few decades. It has been estimated that extrapulmonary tuberculosis comprises about $20 \%$ of all tuberculosis cases. Nevertheless, thyroid tuberculosis (TT) is quite a rare disease even in countries where the prevalence of tuberculosis is high. In the literature, it has been reported that the mean age at onset of TT is around the fourth decade with a slight female preponderance (1).

Extrathyroidal involvements of the disease also exist in the majority of cases with TT. Mycobacterium tuberculosis infects the thyroid gland mainly through miliary spread as a part of generalized dissemination or via direct extension from adjacent lymph nodes. However, primary TT has also been reported, although the involvement of thyroid gland without an extrathyroid focus is extremely rare (2). Both clinical and radiological features of TT are nonspecific and variable. TT can manifest itself as a nodule or abscess in the thyroid gland and it can also mimick carcinoma (3). Therefore, a definitive diagnosis often requires a histological examination.

Herein, we aimed to report this case where tuberculosis was detected in both the thyroid gland and cervical lymph nodes after the surgical excision and to review the relevant literature.

\section{CASE REPORT}

A 53-year-old female patient applied to the Otorhinolaryngology department of Gozde Academy Hospital with complaint of a painless swelling on the right side and middle of her neck. She stated that these lumps on her neck had existed for a few years but they had grown in the last months. The patient had no history of chronic illness except well-controlled hypertension which was being treated by olmesartan 20 milligram per day. Her family history was unremarkable. She had no history of any

Corresponding Author: Ahmet Görgel E-mail: ahmetgorgel@gmail.com

Submitted: 03.12.2020 • Accepted: 03.06.2021

This work is licensed under Creative Commons Attribution-NonCommercial 4.0 International License. 
known contact with any individuals with tuberculosis. There was also no complaints of fever, night sweats, anorexia, weight loss, cough or hemoptysis. On physical examination, multiple solid masses, the largest of them being approximately $3 \mathrm{~cm}$ in size, were detected on the right cervical and supraclavicular region. The masses were round-shaped and they had smooth surfaces and well-defined margins. The overlying skin appeared normal without signs of inflammation and there was no tenderness on palpation. The regional examination did not show any abnormalities except multinodular goiter and other systemic examinations were also normal.

The neck ultrasonography revealed multiple, variable size, hyperechoic solid nodules in both thyroid lobes. The largest of the nodules was $3 \times 2 \mathrm{~cm}$ in size in the left lobe of the thyroid gland and some of the nodules have internal calcification. Magnetic resonance imaging (MRI) of the neck showed that multiple nodular lesions in the thyroid gland were mildly hyperintense on T2-weighted images (Figure 1). In addition, multiple lymphadenopathies that were strongly hyperintense on T2-weighted images on the right cervical (Figure 2) and the right supraclavicular region (Figure 3 ) that were showing diffuse contrast agent uptake after intravenous injection were detected (an informed consent was taken for the images from the patient).

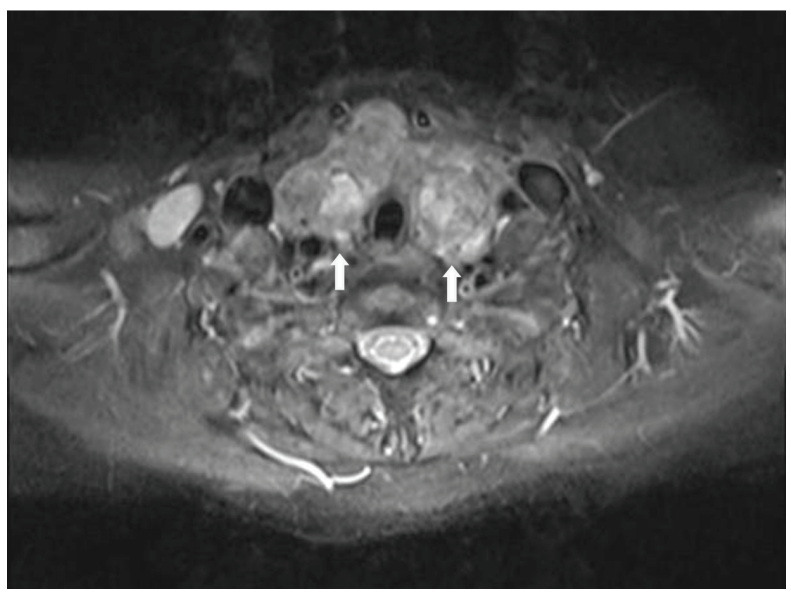

Figure 1: Multiple calcified nodular lesions in both thyroid lobes which seem mildly hyperintense on T2-weighted image on magnetic resonance imaging of the neck (white arrows)

In the laboratory examinations, triiodothyronine, thyroxine and thyroid stimulating hormone levels were all within normal limits. Routine blood investigations showed that haemoglobin was $13.9 \mathrm{~g} / \mathrm{dL}$, and the white blood cells count was $6.3 \mathrm{x}$ $10^{3} / \mathrm{mm}^{3}$ with neutrophils comprising $72 \%$. The erythrocyte sedimentation rate was $4 \mathrm{~mm} / \mathrm{hr}$. Coagulation profile, fasting glucose, hepatic and renal function tests were normal. The human immunodeficiency virus (HIV) status of the patient was negative. The chest X-ray and electrocardiogram were also unremarkable. Additionally, the result of a fine-needle aspiration biopsy (FNAB) that had been performed in another clinic three weeks ago was nondiagnostic.

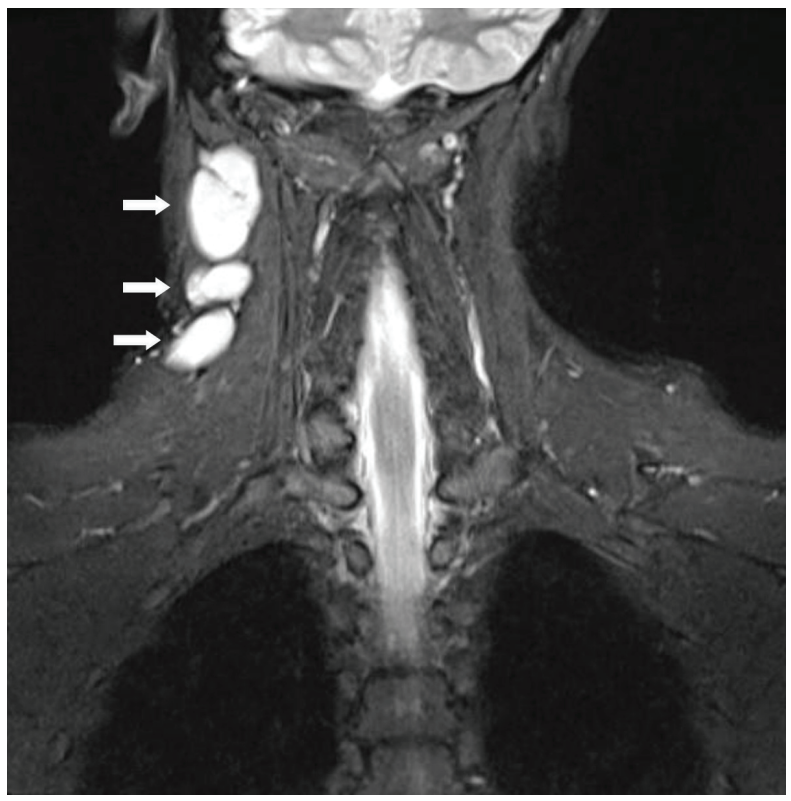

Figure 2: Multiple enlarged lymph nodes that appear strongly hyperintense on T2-weighted coronal image on the right cervical region (horizontal arrows)

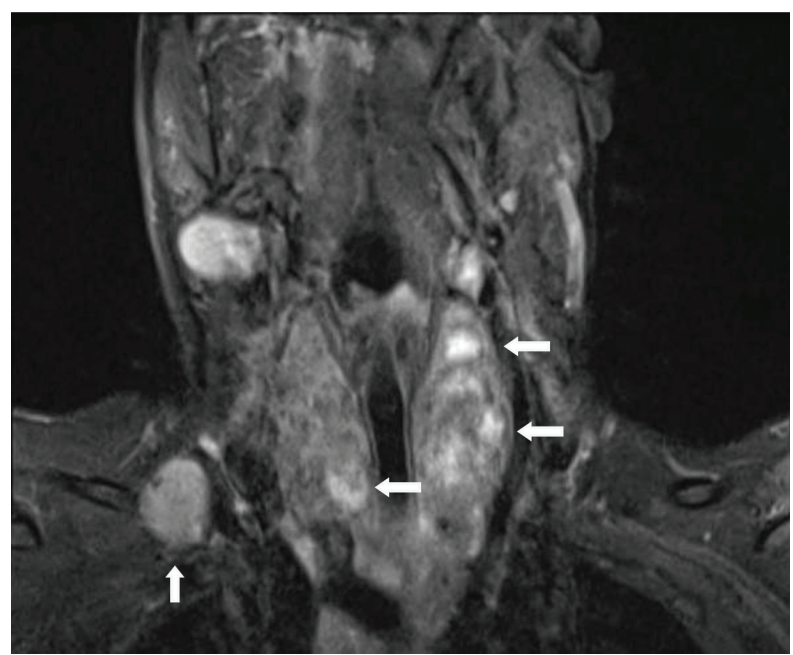

Figure 3: An enlarged lymph node that seems hyperintense on T2-weighted coronal image on the right supraclavicular region (vertical arrow) in addition to multiple calcified nodular lesions in both thyroid lobes (horizontal arrows)

In the light of all these findings, we decided to perform surgery to achieve the final diagnosis because our case was highly suspecious in terms of the possibility of a thyroid carcinoma with metastatic cervical lymphadenopathies. Furthermore, this possibility could not have been ruled out although FNAB had also been made. Total thyroidectomy and right modified neck dissection were performed. Both the thyroid gland and all of the pathological lymph nodes on the neck were excised without any surgical complications (Figure 4), thereafter levothyroxine therapy (100 micrograms per day) was started. 


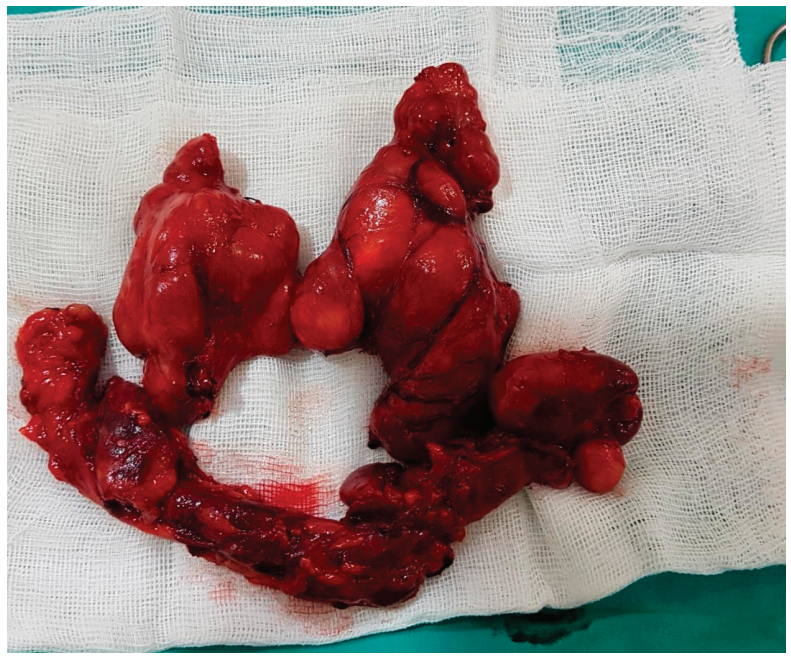

Figure 4: Surgical specimen consisting of the thyroid gland and conglomerate multiple lymph nodes

Histopathological examination unexpectedly showed that the lesions on both the thyroid gland and the extracted lymph nodes consisted of tuberculosis granulomas with caseating necrosis (Figure 5, 6). Thereupon, the patient was treated with antituberculosis drugs that consisted of isoniazid, rifampicin and ethambutol for 6 months. There was no disease recurrence in the course of 15 months clinical follow-up.
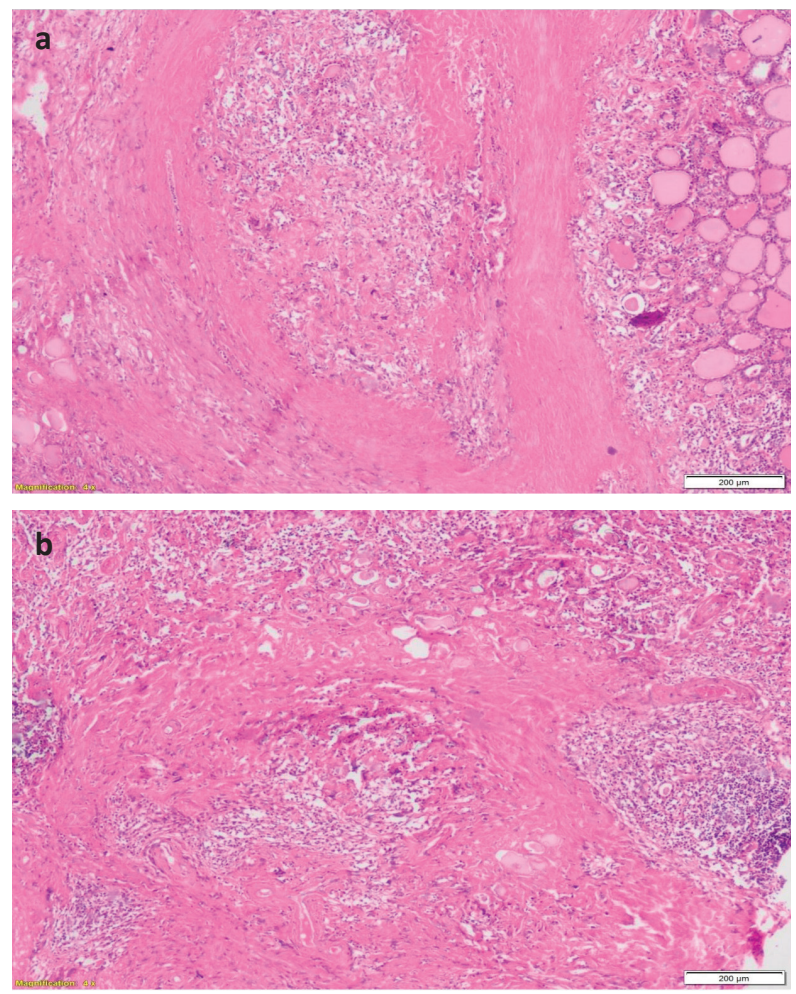

Figure 5: Histopathological view consistent with tuberculosis of the thyroid gland

a) Granulomatous structuring by inflammatory cell infiltration accompanied by atrophic follicles

b) The demonstration of caseating necrosis, epithelioid cell granulomas and Langhans' giant cells

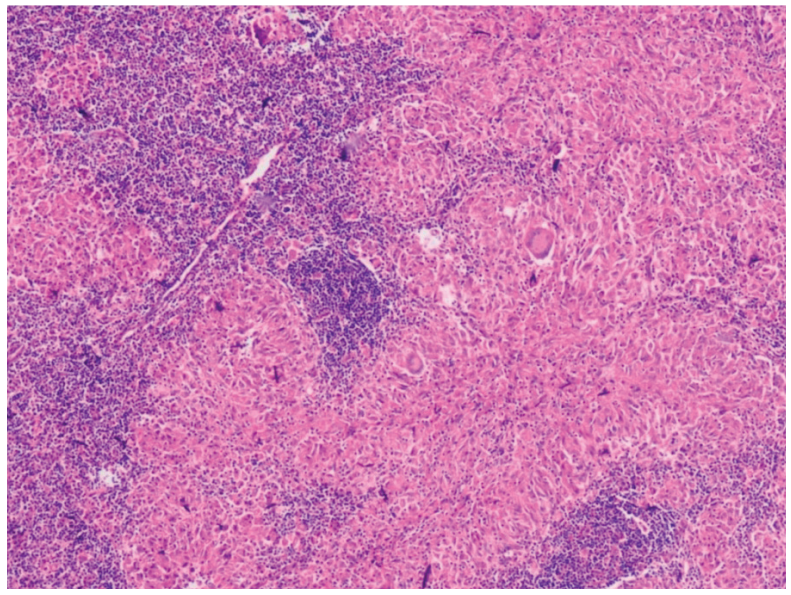

Figure 6: Tuberculous lymphadenitis characterized with caseified granulomas and Langhans' cells

\section{DISCUSSION}

TT is a rare disease whose exact prevalence is not known due to the diagnostic difficulties. The incidence is about $1 \%$, even in developing countries $(4,5)$. However, it has been reported in $2-7 \%$ of cases during autopsy (6). Although the incidence has slightly risen in recent years, probably due to the increased use of FNAB in diagnosis of various thyroid lesions, TT still remains one of the rare forms of extrapulmonary tuberculosis. It has been suggested that the thyroid gland has some natural barriers against invasion by Mycobacterium tuberculosis, such as, high blood flow and rich iodine content of the gland as well as bactericidal action of colloid material inside the thyroid follicles. However, certain risk factors, such as advanced age, malnutrition, diabetes mellitus, and other immunocompromised conditions may create a susceptibility to development of tuberculosis. It has been reported that the frequency of TT is quite high in patients with HIV-induced immunosuppression (7).

TT mostly occurs due to the haematogenous dissemination of bacteria from elsewhere in the body, however, direct spread from the adjacent foci such as the larynx, the trachea and cervical or mediastinal lymph nodes may also be possible. Primary involvement of the thyroid gland has been reported as being extremely rare $(8,9)$. Although tuberculous was revealed both in the thyroid gland and in the lymph nodes, we were not able to determine which one of them was the primary tuberculous focus in our case.

The diagnosis of TT is difficult because it has no specific clinical properties, therefore symptomatology alone typically cannot achieve diagnostic accuracy in this disease. TT usually leads to focal caseous thyroiditis that is limited in the gland, however it may also present itself as localized swelling, diffuse or nodular goiter, cold abscess and (rarely), acute abscess. More importantly, TT may mimic thyroid carcinoma especially if the conglomerate cervical lymph nodes accompany it.

Thyroid function tests are normal in the majority of the cases with TT although a few cases have been reported associated 
with thyrotoxicosis $(9,10)$. Thyrotoxicosis may also be a transient period preceding hypothyroidism, as in the course of subacute thyroiditis. Persistent hypothyroidism generally develops as a result of the inflammatory destruction of the thyroid gland and subsequently the replacement of thyroid follicles with fibrous tissue.

Imaging techniques are not very helpful in diagnosis either. Neck ultrasounds may reveal round heterogeneously hypoechoic unique or multiple lesions or anechoic lesion, irregular borders of nodules, and regional adenopathy (11). Contrary to what is mentioned above, multiple hyperechoic nodules were present in our case. Computed tomography findings of TT are also mostly non-specific. Madhusudhan et al. (12) have reported that the tuberculous lesion in the thyroid gland of their case showed an intermediate signal on T2weighted images on MRI, the signal intensity was higher than the normal glandular parenchyma. Likewise, the MRI images of our case were similar to theirs. However, this appearance in the thyroid gland is not specific, and thyroid carcinoma can also have a similar feature (13).

FNAB is an effective and inexpensive method to diagnose both TT and other thyroid lesions. Acid-fast bacilli staining and culture from fine needle aspiration material are among the most reliable diagnostic methods. However, it is reported in the literature that the acid-fast bacilli are not always demonstrable in the lesion (14). Hence, the diagnosis of TT is frequently delayed and may represent an incidental finding at pathological examination (15). Some authors adopt the definitive histological and bacteriological evidence as the diagnostic essential criterion $(16,17)$. Histopathological features include caseating necrosis, epithelioid cell granulomas and Langhans' giant cells. Furthermore, epithelioid granulomas may be seen in the course of not only TT but also other granulomatous inflammatory processes such as sarcoidosis, subacute thyroiditis, fungal infections, syphilis, thyroid neoplasia, granulomatous vasculitis, and foreign body reaction. However, the demonstration of acid-fast bacilli and the presence of caseating necrosis are pathognomonic for tuberculous thyroiditis. In this case, we preferred surgery without repeating FNAB because the findings were highly suspicious in point of malignancy. However, the findings may have been misleading as also seen in our case. Nevertheless, we considered that the operation was not a completely unnecessary attempt for the patient. We could have missed the presence of the tuberculosis or a possible malignancy even if FNAB had been repeated before surgery. TT may mistakenly be thought to be thyroid carcinoma. Moreover, TT might also coexist with thyroid carcinoma (18, 19). Treatment of TT is primarily based on antituberculous medications and where the response to antituberculous therapy is satisfactory. A thyroidectomy should be performed on large masses, TT cases coexisting with thyroid carcinoma or mimicking malignant lesions (20).

As a consequence, TT is a rare entity and it may resemble thyroid carcinoma in the first evaluation. Therefore, it should be taken into account in the differential diagnosis of thyroid lesions and a careful approach should be adopted to avoid unnecessary surgical intervention.

Peer Review: Externally peer-reviewed.

Informed Consent: Informed consent was taken for the images from the patient.

Author Contributions: Conception/Design of Study- E.K., A.G.; Data Acquisition- Ş.F.E., A.A.; Data Analysis/Interpretation- A.G., Ş.F.E.; Drafting Manuscript- A.G., Ş.F.E.; Critical Revision of Manuscript- E.K., A.A.; Final Approval and Accountability- A.G., E.K., Ş.F.E., A.A.

Conflict of Interest: Authors declared no conflict of interest.

Financial Disclosure: Authors declared no financial support.

\section{REFERENCES}

1. Bulbuloglu E, Ciralik H, Okur E, Ozdemir G, Ezberci, F, Cetinkaya A. Tuberculosis of the Thyroid Gland: Review of the Literature. World J Surg 2006;30:149-55.

2. Akbulut S, Sogutcu N, Arikanoglu Z, Bakir S, Ulku A, Yagmur Y. Thyroid tuberculosis in southeastern Turkey: is this the resurgence of a stubborn disease? World J Surg 2011;35:1847-52.

3. Balasarkar D, Dhareswar J, Satoskar RR, Awsare N, Mahey R, Kumar V. Primary thyroid tuberculosis. Available from: URL: https://www. bhj.org.in/journal/special_issue_tb/SP_13.HTM

4. Mondal A, Patra DK. Efficacy of fine needle aspiration cytology in the diagnosis of tuberculosis of the thyroid gland: a study of 18 cases. J Laryngol Otol 1995;109:36-8.

5. Ozekinci S, Mizrak B, Saruhan G, Senturk S. Histopathologic diagnosis of thyroid tuberculosis. Thyroid 2009;19(9):983-6.

6. Simkus A. Thyroid tuberculosis. Medicina (Kaunas) 2004;40:201-4.

7. Basílio-De-Oliveira CA. Infectious and neoplastic disorders of the thyroid in AIDS patients: an autopsy study. Braz J Infect Dis 2000; 4(2):67-75.

8. Pazaitou K, Chrisoulidou A, Ginikopoulou E, Angel J, Destouni C, Vainas I. Primary tuberculosis of the thyroid gland: report of three cases. Thyroid 2002;12(12):1137-40.

9. Dhar R, Rana M, Bhardwaj S. Primary Tubercular Granulomatous Thyroiditis, Presenting as Thyroid Nodule with Hyperthyroidism, Pyrexia of Unknown Origin and Severe Anemia. J Assoc Physicians India 2018;66(5):94-5.

10. Kapoor VK, Subramani K, Das SK, Mukhopadhyay AK, Chattopadhyay, TK. Tuberculosis of the thyroid gland associated with thyrotoxicosis. Postgrad Med J 1985;61:339-40.

11. Oueslati I, Sakka I, Ismail O, Akrout I, Marghli A, Chihaoui M. Tuberculosis of the Thyroid Gland Presented as a Rapid Enlargement of a Preexisting Goiter. Case Reports in Endocrinology 2018;10:1-4.

12. Madhusudhan KS, Seith A, Khadgawat R, Das P, Mathur S. Tuberculosis of the thyroid gland: magnetic resonance imaging appearances. Singapore Med J 2009;50(7):235-8.

13. Gotway $M B$, Higgins $C B$. MR imaging of the thyroid and parathyroid glands. Magn Reson Imaging Clin N Am 2000;8(1):163-82, ix.

14. Chaurasia JK, Garg C, Agarwal A, Naim M. Tubercular thyroiditis with multinodular goitre with adenomatous hyperplasia: a rare coexistence. BMJ Case Rep 2013; pii: bcr2013200861. doi: 10.1136/bcr-2013-200861. 
15. Jiang Y-D, Gao J-Q, Chen S, Deng X-Y. Thyroid tuberculosis: two cases report and review of the literature. Int J Clin Exp Med 2016;9(11):22477-85.

16. Sanehi S, Dravid C, Chaudhary N, Rai AK. Primary thyroid tuberculosis. Indian J Otolaryngol Head Neck Surg 2007;59:154-6.

17. Chandanwale SS, Buch AC, Vimal SS, Sachdeva P. Thyroid tuberculosis: presenting symptom of mediastinal tuberculous lymphadenitis-an unusual case. Indian J Tuberc 2014;61:84-7.
18. Meng L, Hu S, Huang L, Xu C. Papillary thyroid cancer coexisting with thyroid tuberculosis: A case report. Oncol Lett 2014;7(5): 1563-5.

19. Errami N, Benjellounb A, Hemmaouia B, Nadoura K, Benariba F. Association of tuberculous thyroiditis and papillary carcinoma of the thyroid: a rare coincidence. Pan Afr Med J 2014;19:118. doi: 10.11604/pamj.2014.19.118.4805.

20. Zendah I, Daghfous H, Ben Mrad S, Tritar F. Primary tuberculosis of the thyroid gland. Hormones (Athens) 2008;7:330-3. 